Schmerz 2016 · 30:293

DOI 10.1007/s00482-016-0101-1

Online publiziert: 11. Februar 2016

(c) Deutsche Schmerzgesellschaft e.V. Published by Springer-Verlag Berlin Heidelberg - all rights reserved 2016

\section{CrossMark}

\author{
E. M. Pogatzki-Zahnn ${ }^{1} \cdot$ W. Meissner ${ }^{2}$ \\ ${ }^{1}$ Klinik für Anästhesiologie, operative Intensivmedizin und Schmerztherapie, \\ Universitätsklinikum Münster, Münster, Deutschland \\ 2 Klinik für Anästhesiologie und Intensivmedizin, Sektion Schmerztherapie, \\ Universitätsklinikum Jena, Jena, Deutschland
}

\title{
Akutschmerztherapie: nicht auf dem Erreichten ausruhen
}

ist es, sich deshalb an die ersten Stunden zu erinnern (oder erinnern zu lassen), um sich bewusst zu machen, wie viel sich in den letzten Jahrzehnten doch getan hat. Die deutsche Pionierarbeit wird auch deshalb oft unterschätzt, weil sich viele der damaligen Aktivitäten (noch) nicht in internationalen Journalen widerspiegelten.

Für die Zukunft hat dies ebenfalls bedeutende Implikationen. So haben doch der letzte Zensus [1] und die Datenauswertungen der Qualitätsverbesserung in der postoperativen Schmerztherapie (QUIPS; [2, 3]) gezeigt, dass die deutsche ASD-Landschaft immer noch ausbauwürdig ist; die Qualität der Akutschmerztherapie in deutschen Krankenhäusern darf nicht durch wirtschaftliche oder andere Zwänge eingeengt, sondern muss gesichert und gestärkt werden. Hierbei kann unser Fach, die Anästhesiologie, eine entscheidende Rolle spielen - aber auch ein klares Bekenntnis zu diesem Teilgebiet abgeben und entsprechende Unterstützung bereitstellen! Möglicherweise kann darüber hinaus die Einführung generischer Qualitätsindikatoren im Bereich der Schmerzmedizin die Versorgung der Patienten weiter verbessern.

Rund 40 Jahre nach Einführung der ersten ASD hat sich viel getan - aber dies ist kein Grund, sich auf dem Erreichten auszuruhen. C. Maier, H. Wulf, K.A. Lehmann, W. Seeling, U.M. Stamer und weitere Pioniere der Akutschmerztherapie haben uns dazu den Weg gewiesen.

\section{Korrespondenzadresse}

Prof. Dr. E. M. Pogatzki-Zahn

Klinik für Anästhesiologie, operative Intensivmedizin und Schmerztherapie, Universitätsklinikum Münster

Albert-Schweitzer-Campus 1, Gebäude A1, 48149 Münster

pogatzki@anit.uni-muenster.de

Interessenkonflikt. E. M. Pogatzki-Zahn und W. Meissner geben an, dass kein Interessenkonflikt besteht.

\section{Literatur}

1. Erlenwein J, Stamer U, Koschwitz R, Koppert W, Quintel M, Meißner W, Petzke F (2014) Akutschmerztherapie in der stationären Versorgung an deutschen Krankenhäusern: Ergebnisse des Akutschmerzzensus 2012. Schmerz 28:147-156

2. Gerbershagen HJ, Aduckathil S, van Wijck AJ, Peelen LM, Kalkman CJ, Meissner W (2013) Pain intensity on the first day after surgery: a prospective cohort study comparing 179 surgical procedures. Anesthesiology 118(4):934-944

3. Gerbershagen HJ, Pogatzki-Zahn E, Aduckathil S, Peelen LM, Kappen TH, van Wijck AJ, Kalkman CJ, Meissner W (2014) Procedure-specific risk factor analysis for the development of severe postoperative pain. Anesthesiology 120(5):1237-1245

4. Maier C, Kibbel K, Mercker S, Wulf H (1994) Postoperative Schmerztherapie auf Allgemeinen Pflegestationen - Analyse der achtjährigen Tätigkeit eines Anästhesiologischen Akut-Schmerzdienstes. Anaesthesist 43:385-397

5. Ready LB, Oden R, Chadwick HS, Benedetti C, Rooke GA, Caplan R, Wild LM (1988) Development of an anesthesiology-based postoperative pain management service. Anesthesiology 68(1):100106 seine Runden dreht - sofern man in einer der etwa $50 \%$ deutschen Kliniken arbeitet, die einen solchen Dienst haben -, sind echte Konflikte mit chirurgischen oder anästhesiologischen Kollegen doch eher die Ausnahme. Umso wesentlicher 